\title{
A DIRECT APPROACH TO DERIVING FILTERING EQUATIONS FOR DIFFUSION PROCESSES
}

\author{
N. V. KRYLOV AND A. ZATEZALO
}

\begin{abstract}
Filtering equations are derived for conditional probability density functions in case of partially observable diffusion processes by using results and methods from the $L_{p}$-theory of SPDEs. The method of derivation is new and does not require any knowledge of filtering theory.
\end{abstract}

\section{IntRoduCtion}

The main purpose of the article is to give a derivation of the so-called filtering equation for partially observable diffusion processes on the basis of the $L_{p}$-theory of SPDE's from [5] without using any facts from filtering theory itself. We refer the reader to [11] for a standard derivation, bibliography, and historical remarks.

As in [7], where finite-state Markov processes are considered, we follow a "direct" PDE approach as opposed to purely probabilistic one. To give an idea of what we mean by these approaches, let us consider Itô's stochastic equation

$$
d x_{t}=\sigma\left(x_{t}\right) d w_{t}+b\left(x_{t}\right) d t,
$$

say in one dimension with nonrandom coefficients satisfying appropriate conditions. Let $x_{t}(x)$ be a solution of this equation starting at $x$. Take a smooth bounded function $\phi(x)$ and define $v(t, x)=E \phi\left(x_{t}(x)\right)$. Then under appropriate conditions $v$ satisfies Kolmogorov's equation

$$
D_{t} u=\frac{1}{2} \sigma^{2} u_{x x}+b u_{x} \quad t \geq 0, x \in \mathbb{R}, \quad u(0, x)=\phi(x),
$$

where $D_{t}=\partial / \partial t$. One can derive this fact in two ways. Historically, the first one suggested in [1] is based on proving probabilistically that $v$ is smooth enough and then using Markov property (for detail see, for instance, [3]). In this way Kolmogorov's equation is derived even if the diffusion can degenerate. However if the process is nondegenerate, one can use a "direct" approach consisting of taking the solution $u$ of (1.1), the existence of which is guaranteed by the theory of PDEs, and applying Itô's formula to $u\left(T-t, x_{t}(x)\right)$. Then one gets

$$
u\left(0, x_{T}(x)\right)=u\left(T, x_{0}(x)\right)+\int_{0}^{T} \sigma\left(x_{s}(x)\right) u_{x}\left(x_{s}(x)\right) d w_{s}
$$

and, to prove that $u=v$ and hence $v$ satisfies (1.1), it only remains to take expectations and notice that $u\left(0, x_{T}\right)=\phi\left(x_{T}\right)$ and $x_{0}(x)=x$.

In our derivation of filtering equations we will closely follow the idea behind this second derivation of Kolmogorov's equation. We will take the filtering equation, take its solution, the existence of which is guaranteed this time by the theory from

1991 Mathematics Subject Classification. 60G35,62M20.

Key words and phrases. filtering, diffusion processes, $L_{p}$-theory of SPDE's.

The work of the first author was partially supported by the NSF Grant DMS-9625483. 
[5], and then apply Itô's formula to appropriately chosen functions. Observe that this way of arguing was already used in [10] and [12] to prove the uniqueness of measure-valued solutions to the Zakai equation. Also we notice that in [15] one can find similar derivation of filtering equations based not only on $L_{p}$-theory but also on $C^{2+\alpha}$-theory of parabolic second-order equations developed in [9] for equations with coefficients only measurable in time.

The article is organized as follows. Section 2 contains the statement of the problem and the main result. In Section 3 we explain the main idea of proving Theorem 2.10. Section 4 contains necessary facts from $L_{p}$-theory of SPDEs borrowed from [5] (or [4]). The final Section 5 contains the proof of Theorem 2.10.

\section{Filtering Equation}

Let $(\Omega, \mathcal{F}, P)$ be a complete probability space with an increasing filtration $\left\{\mathcal{F}_{t}, t \geq\right.$ $0\}$ of complete with respect to $P \sigma$-fields $\mathcal{F}_{t} \subset \mathcal{F}$. Denote $\mathcal{P}$ the predictable $\sigma$-field in $\Omega \times(0, \infty)$ associated with $\left\{\mathcal{F}_{t}\right\}$. Let $d, d_{1}>d$, and $d_{2} \geq d_{1}$ be integers and $w_{t}$ be a $d_{2}$-dimensional Wiener process with respect to $\left\{\mathcal{F}_{t}\right\}$. Let $K, T, \delta>0$ be fixed finite constants.

Consider a $d_{1}$-dimensional two component process $z_{t}=\left(x_{t}, y_{t}\right)$ with $x_{t}$ being $d$ dimensional and $y_{t} d_{1}-d$-dimensional. We assume that $z_{t}$ is defined as a solution of the system

$$
\begin{aligned}
& d x_{t}=b\left(t, z_{t}\right) d t+\theta\left(t, z_{t}\right) d w_{t} \\
& d y_{t}=B\left(t, z_{t}\right) d t+\Theta\left(t, y_{t}\right) d w_{t}
\end{aligned}
$$

with some initial data.

The coefficients of (2.1) are assumed to be vector- or matrix-valued functions of appropriate dimensions defined on $[0, T] \times \mathbb{R}^{d_{1}}$. Actually $\Theta(t, y)$ is assumed to be independent of $x$, so that it is a function on $[0, T] \times \mathbb{R}^{d_{1}-d}$ rather than $[0, T] \times \mathbb{R}^{d_{1}}$ but as always we may think of $\Theta(t, y)$ as of function of $(t, z)$ as well. Next we enumerate more technical assumptions.

Assumption 2.1. The functions $b, \theta, B$ and $\Theta$ are Borel measurable and bounded functions of their arguments. Each of them satisfies the Lipschitz condition with constant $K$ with respect to $z$. Moreover $\theta(t, x, y)$ is continuously differentiable with respect to $x$ (not $z$ ) and its first derivatives to respect to $x^{i}$ s satisfy the Lipschitz condition with constant $K$ with respect to $x$ (not $z$ ).

Assumption 2.2. The symmetric matrix $\Theta \Theta^{*}$ is invertible and $\Psi:=\left(\Theta \Theta^{*}\right)^{-\frac{1}{2}}$ is a bounded function of $(t, y)$.

Remark 2.3. From Assumption 2.2 we have that there exists a constant $N$, such that, for any $(t, y) \in[0, T] \times R^{d_{1}-d}$ and $\eta \in R^{d_{1}-d},\left|\left(\Theta \Theta^{*}\right)^{-\frac{1}{2}}(t, y) \eta\right|^{2} \leq N|\eta|^{2}$. Defining $\xi:=\left(\Theta \Theta^{*}\right)^{-\frac{1}{2}}(t, y) \eta$, we find $|\xi|^{2} \leq N\left(\Theta \Theta^{*}(t, y) \xi, \xi\right)$ which shows that $\Theta \Theta^{*}$ is uniformly positive definite.

Assumption 2.4. For any $\xi \in R^{d}, z=(x, y) \in R^{d_{1}}$, and $t>0$,

$$
\left|Q(t, y) \theta^{*}(t, z) \xi\right|^{2} \geq \delta|\xi|^{2},
$$

where $Q$ is the orthogonal projector on $\operatorname{Ker} \Theta$. In other words

$$
\left(\theta\left(I-\Theta^{*} \Psi^{2} \Theta\right) \theta^{*} \xi, \xi\right) \geq \delta|\xi|^{2}
$$


Remark 2.5. From (2.2) we see that $\theta \theta^{*}$ is uniformly positive definite with constant of positivity $\delta$.

In the following assumption and everywhere in the article by $H_{p}^{\gamma}=H_{p}^{\gamma}\left(\mathbb{R}^{d}\right)$ we denote the space of Bessel potentials (see, for instance, [14]).

Assumption 2.6. The random vectors $x_{0}$ and $y_{0}$ are independent of the process $w_{t}$. The conditional distribution of $x_{0}$ given $y_{0}$ has a density, which we denote by $\pi_{0}(x)=\pi_{0}(\omega, x)$. We have $\pi_{0} \in L_{p}\left(\Omega, H_{p}^{2-2 / p}\right)$.

Next we introduce few notation. Let

$$
\begin{gathered}
\Psi_{t}=\Psi\left(t, y_{t}\right), \quad \Theta_{t}=\Theta\left(t, y_{t}\right), \quad a_{t}(x)=\frac{1}{2} \theta \theta^{*}\left(t, x, y_{t}\right), \quad b_{t}(x)=b\left(t, x, y_{t}\right) \\
\sigma_{t}(x)=\theta\left(t, x, y_{t}\right) \Theta_{t}^{*} \Psi_{t}, \quad \beta_{t}(x)=\Psi_{t} B\left(t, x, y_{t}\right) \\
L_{t}(x)=a_{t}^{i j}(x) \frac{\partial^{2}}{\partial x^{i} \partial x^{j}}+b_{t}^{i}(x) \frac{\partial}{\partial x^{i}} \\
L_{t}^{*}(x) u(t, x)=\frac{\partial^{2}}{\partial x^{i} \partial x^{j}}\left(a_{t}^{i j}(x) u(t, x)\right)-\frac{\partial}{\partial x^{i}}\left(b_{t}^{i}(x) u(t, x)\right) \\
\Lambda_{t}^{k}(x) u(t, x)=\beta_{t}^{k}(x) u(t, x)-\frac{\partial}{\partial x^{i}}\left(\sigma_{t}^{i k}(x) u(t, x)\right) \\
\tilde{\theta}_{t}(z)=\left(\begin{array}{c}
\theta(t, z) \\
\Theta(t, y)
\end{array}\right), \quad \tilde{a}_{t}(z)=\frac{1}{2} \tilde{\theta} \tilde{\theta}_{t}^{*}(z), \quad \tilde{b}_{t}(z)=\left(\begin{array}{c}
b(t, z) \\
B(t, z)
\end{array}\right) \\
\tilde{L}_{t}(z)=\tilde{a}_{t}^{i j}(z) \frac{\partial^{2}}{\partial z^{i} \partial z^{j}}+\tilde{b}_{t}^{i}(z) \frac{\partial}{\partial z^{i}}
\end{gathered}
$$

where $t \in[0, T], x \in R^{d}, k=1, \ldots, d_{1}-d$, and we use the summation convention over all "reasonable" values of repeated indices, so that the summation in (2.3), (2.4), and (2.5) is done for $i, j=1, \ldots, d$ and in (2.7) for $i, j=1, \ldots, d_{1}$.

Finally, by $\mathcal{F}_{t}^{y}$ we denote the completion of $\sigma\left\{y_{s}: s \leq t\right\}$ with respect to $P, \mathcal{F}$.

Remark 2.7. System of equations (2.1) can be now written as

$$
d z_{t}=\tilde{b}\left(t, z_{t}\right) d t+\tilde{\theta}\left(t, z_{t}\right) d w_{t} .
$$

Remark 2.8. From Remark 2.5 we have that the operator $L$ defined by (2.3) is uniformly elliptic with constant of ellipticity $\delta$. It turns out that, in a sense, Assumptions 2.4 and 2.2 are equivalent to uniform nondegeneracy of the process $z_{t}$ or to uniform nondegeneracy of the operator $\tilde{L}$ defined by (2.7).

To show this, we take $\zeta=(\xi, \eta) \in R^{d} \times R^{d_{1}-d}$ and observe that

$$
\begin{aligned}
2(\tilde{a} \zeta, \zeta)=\left(\tilde{\theta} \tilde{\theta}^{*} \zeta, \zeta\right)= & \left(\theta \theta^{*} \xi, \xi\right)+2\left(\Theta \theta^{*} \xi, \eta\right)+\left(\Theta \Theta^{*} \eta, \eta\right) \\
& =\left(\theta \theta^{*} \xi, \xi\right)+2\left(\Psi \Theta \theta^{*} \xi, \tilde{\eta}\right)+\varepsilon(\tilde{\eta}, \tilde{\eta})+(1-\varepsilon)\left(\Theta \Theta^{*} \eta, \eta\right)
\end{aligned}
$$

where for simplicity of notation we drop obvious values of arguments, $\tilde{\eta}=\Psi^{-1} \eta$, and $\varepsilon \in(0,1)$. By using $2(\mu, \nu)+\varepsilon|\mu|^{2} \geq-\varepsilon^{-1}|\nu|^{2}$ and taking $N$ from Remark 2.3, we see that

$$
\begin{gathered}
2(\tilde{a} \zeta, \zeta) \geq\left(\theta \theta^{*} \xi, \xi\right)-\varepsilon^{-1}\left(\Psi \Theta \theta^{*} \xi, \Psi \Theta \theta^{*} \xi\right)+(1-\varepsilon) N^{-1}|\eta|^{2} \\
\geq \delta|\xi|^{2}+\left(1-\varepsilon^{-1}\right)\left(\Psi \Theta \theta^{*} \xi, \Psi \Theta \theta^{*} \xi\right)+(1-\varepsilon) N^{-1}|\eta|^{2}
\end{gathered}
$$


where the last inequality follows from (2.2). Finally, $\Psi \Theta \theta^{*}$ is a bounded function, so that, for a constant $N_{1}$,

$$
2(\tilde{a} \zeta, \zeta) \geq\left(\delta+N_{1}\left(1-\varepsilon^{-1}\right)\right)|\xi|^{2}+(1-\varepsilon) N^{-1}|\eta|^{2} .
$$

For $\varepsilon$ sufficiently close to 1 the last expression is greater than $\delta_{1}|\zeta|^{2}$ with a constant $\delta_{1}>0$, which is equivalent to uniform ellipticity of $\tilde{a}$.

Let us consider the following initial value problem

$$
\begin{gathered}
d \bar{\pi}_{t}(x)=L_{t}^{*}(x) \bar{\pi}_{t}(x) d t+\Lambda_{t}^{k}(x) \bar{\pi}_{t}(x) \Psi_{t}^{k r} d y_{t}^{r}, \\
\bar{\pi}_{0}(x)=\pi_{0}(x),
\end{gathered}
$$

where $t \in[0, T], x \in \mathbb{R}^{d}$ and $\bar{\pi}_{t}(x)=\bar{\pi}_{t}(\omega, x)$. We will be looking for solutions $\bar{\pi}$ belonging to the space $\mathcal{H}_{p}^{2}(T)$, which is introduced in [5] or [4] and described in much detail in our Section 4. Also the sense in which we understand (2.9) is described there.

Lemma 2.9. Let Assumptions 2.1-2.6 be satisfied. Then in $\mathcal{H}_{p}^{2}(T)$ there exists a unique solution $\bar{\pi}$ of (2.9). In addition $\bar{\pi}_{t} \geq 0$ for all $t \in[0, T]$ (a.s).

The proof of this lemma is given in Section 4 .

We intend to give a direct proof of the following theorem which is proved in a different manner in Section 8 of [5]. By the way, Assumption 2.1 looks a little bit weaker than the corresponding assumption in [5]. The fact that they are indeed equivalent is discussed before Lemma 8.2 of [5].

Theorem 2.10. Let $\bar{\pi}$ be the function from Lemma 2.9. Then $0<\int_{\mathbb{R}^{d}} \bar{\pi}_{t}(x) d x<$ $\infty$ for all $t \in[0, T]$ (a.s.) and for any real-valued, bounded or nonnegative, (Borel) measurable function $f$ given on $\mathbb{R}^{d}$

$$
E\left[f\left(x_{t}\right) \mid \mathcal{F}_{t}^{y}\right]=\frac{\int_{\mathbb{R}^{d}} f(x) \bar{\pi}_{t}(x) d x}{\int_{\mathbb{R}^{d}} \bar{\pi}_{t}(x) d x} \quad \text { (a.s.). }
$$

\section{The IDEA OF THE PROOF}

Let $z_{t}$ be the solution of (2.8) with initial data $z_{0}=\left(x_{0}, y_{0}\right)$. Denote

$$
\begin{gathered}
\tilde{\beta}_{t}=\beta_{t}\left(x_{t}\right), \quad \rho_{t}=\exp \left(-\int_{0}^{t} \tilde{\beta}_{s}^{k} \Psi_{s}^{k r} \Theta_{s}^{r n} d w_{s}^{n}-\frac{1}{2} \int_{0}^{t}\left|\tilde{\beta}_{s}\right|^{2} d s\right), \\
\bar{\rho}_{t}=E\left[\rho_{t} \mid \mathcal{F}_{t}^{y}\right] .
\end{gathered}
$$

Instead of (2.10) we are going to explain how to prove

$$
E\left[f\left(x_{t}\right) \mid \mathcal{F}_{t}^{y}\right]=\bar{\rho}_{t} \int_{\mathbb{R}^{d}} f(x) \bar{\pi}_{t}(x) d x \quad \text { (a.s.). }
$$

This is sufficient because for $f \equiv 1$, it follows from (3.2) that (a.s.)

$$
\bar{\rho}_{t}=\left(\int_{\mathbb{R}^{d}} \bar{\pi}_{t}(x) d x\right)^{-1} .
$$

Let $v_{t}(z)$ be a solution of the deterministic problem

$$
\begin{aligned}
D_{t} v_{t}(z)+\tilde{L}_{t} v_{t}(z)-c_{t}(y) v_{t}(z) & =0 \quad t \in[0, T], z \in \mathbb{R}^{d_{1}}, \\
v_{T}(z) & =\varphi(z) \quad z \in \mathbb{R}^{d_{1}},
\end{aligned}
$$


where $\varphi \in C_{0}^{\infty}\left(\mathbb{R}^{d_{1}}\right), c_{t}(y)$ is a smooth bounded and nonnegative function on $[0, T] \times$ $\mathbb{R}^{d_{1}-d}$, and $D_{t}=\partial / \partial t$. By Itô's formula the process $v_{t}\left(z_{t}\right) \exp \left(-\int_{0}^{t} c_{s}\left(y_{s}\right) d s\right)$ is a (local) martingale on $[0, T]$. In particular (if it is a martingale),

$$
v_{t}\left(z_{t}\right)=E\left[v_{T}\left(z_{T}\right) e^{-\int_{t}^{T} c_{s}\left(y_{s}\right) d s} \mid \mathcal{F}_{t}\right]=E\left[\varphi\left(z_{T}\right) e^{-\int_{t}^{T} c_{s}\left(y_{s}\right) d s} \mid \mathcal{F}_{t}\right]
$$

Hence (3.2) should imply that

$$
\begin{gathered}
E\left[v_{t}\left(z_{t}\right) \mid \mathcal{F}_{t}^{y}\right]=\bar{\rho}_{t} \int_{\mathbb{R}^{d}} v_{t}\left(x, y_{t}\right) \bar{\pi}_{t}(x) d x \\
\mu_{t}:=E\left[\varphi\left(z_{T}\right) e^{-\int_{t}^{T} c_{s}\left(y_{s}\right) d s} \mid \mathcal{F}_{t}^{y}\right]=\bar{\rho}_{t} \int_{\mathbb{R}^{d}} v_{t}\left(x, y_{t}\right) \bar{\pi}_{t}(x) d x=: \nu_{t} .
\end{gathered}
$$

It turns out that $\bar{\pi}_{t}$ is $\mathcal{F}_{t}^{y}$-adapted, so that $\nu_{t}$ is $\mathcal{F}_{t}^{y}$-measurable. The same holds for $\mu_{t}$. Therefore, by multiplying both parts of (3.5) by $\exp \left(-\int_{0}^{t} c_{s}\left(y_{s}\right) d s\right)$ and using definition (3.1) we get

$$
E \varphi\left(z_{T}\right) e^{-\int_{0}^{T} c_{s}\left(y_{s}\right) d s}=E \rho_{t} e^{-\int_{0}^{t} c_{s}\left(y_{s}\right) d s} \int_{\mathbb{R}^{d}} v_{t}\left(x, y_{t}\right) \bar{\pi}_{t}(x) d x
$$

Now comes our main idea. We are going to show that (3.6) holds by showing that the right-hand side is independent of $t$. Observe that its value at time $t=T$ is

$$
E e^{-\int_{0}^{T} c_{s}\left(y_{s}\right) d s} \rho_{T} \int_{\mathbb{R}^{d}} \varphi\left(x, y_{T}\right) \bar{\pi}_{T}(x) d x
$$

whereas at $t=0$, due to the definition of $\pi_{0}$ and formula (3.4), it is equal to

$$
\begin{gathered}
E \int_{\mathbb{R}^{d}} v_{0}\left(x, y_{0}\right) \pi_{0}(x) d x=E E\left[v_{0}\left(z_{0}\right) \mid \mathcal{F}_{0}^{y}\right] \\
=E E\left[\varphi\left(z_{T}\right) e^{-\int_{0}^{T} c_{s}\left(y_{s}\right) d s} \mid \mathcal{F}_{0}^{y}\right]=E \varphi\left(z_{T}\right) e^{-\int_{0}^{T} c_{s}\left(y_{s}\right) d s .}
\end{gathered}
$$

Thus, if the right-hand side of (3.6) is independent of $t$, then (3.6) holds and

$$
E e^{-\int_{0}^{T} c_{s}\left(y_{s}\right) d s} \varphi\left(z_{T}\right)=E e^{-\int_{0}^{T} c_{s}\left(y_{s}\right) d s} \rho_{T} \int_{\mathbb{R}^{d}} \varphi\left(x, y_{T}\right) \bar{\pi}_{T}(x) d x .
$$

It is easy to understand that one can put here indicator of any set $\left\{y_{t_{1}} \in \Gamma_{1}, \ldots, y_{t_{n}} \in\right.$ $\left.\Gamma_{n}\right\}$ in place of $\exp \left(-\int_{0}^{T} c_{s}\left(y_{s}\right) d s\right)$, where $t_{i} \leq T$ and $\Gamma_{i}$ are Borel, and then one immediately obtains

$$
E\left[\varphi\left(z_{T}\right) \mid \mathcal{F}_{T}^{y}\right]=\bar{\rho}_{T} \int_{\mathbb{R}^{d}} \varphi\left(x, y_{T}\right) \bar{\pi}_{T}(x) d x \quad \text { (a.s.). }
$$

This gives (3.2) for $t=T$, which is of course enough.

Proving the independence of the right-hand side of (3.6) of $t$ is done by showing that

$$
\xi_{t}:=\rho_{t} e^{-\int_{0}^{t} c_{s}\left(y_{s}\right) d s} \int_{\mathbb{R}^{d}} v_{t}\left(x, y_{t}\right) \bar{\pi}_{t}(x) d x=\rho_{t} e^{-\int_{0}^{t} c_{s}\left(y_{s}\right) d s}\left(\bar{\pi}_{t}, v_{t}\left(\cdot, y_{t}\right)\right)
$$

is an $\mathcal{F}_{t}$-martingale. By the way so far, we did not use the explicit formula for $\rho_{t}$ or explicit form of the equation defining $\bar{\pi}$. As a matter of fact, they could even be found from the condition that the process $\xi_{t}$ be a martingale. 
Let us give a sketch of the proof that (3.9) is a martingale. The main ingredients are, of course, Itô's formula and integration by parts. The justification of the following argument is given in Section 5. By Itô's formula

$$
\begin{aligned}
& d v_{t}\left(x, y_{t}\right)=v_{t y^{i}}\left(x, y_{t}\right) \Theta_{t}^{i k} d w_{t}^{k} \\
& +\left[D_{t} v_{t}\left(x, y_{t}\right)+\tilde{a}_{t}^{d+i, d+j}\left(y_{t}\right) v_{t y^{i} y^{j}}\left(x, y_{t}\right)+\tilde{b}_{t}^{d+i}\left(x, y_{t}\right) v_{t y^{i}}\left(x, y_{t}\right)\right] d t
\end{aligned}
$$

(where the summation is understood for $i, j=1, \ldots, d_{1}-d$, and $k=1, \ldots, d_{2}$ ). We rewrite this equality by using (3.3), defining

$$
\tilde{w}_{t}=\int_{0}^{t} \Psi_{s} \Theta_{s} d w_{s}, \quad \Phi_{t}=\Psi_{t}^{-1}
$$

and noticing that $\tilde{b}_{t}^{d+i}\left(z_{t}\right)=B^{i}\left(t, z_{t}\right)=\Phi_{t}^{i k} \tilde{\beta}_{t}^{k}$. By the way, observe that, as easily follows from Lévy's theorem, $\left(\tilde{w}_{t}, \mathcal{F}_{t}\right)$ is a $d_{1}-d$-dimensional Wiener process. Then we get that

$$
\begin{aligned}
d v_{t}\left(x, y_{t}\right)=v_{t y^{i}}\left(x, y_{t}\right) \Phi_{t}^{i k} d \tilde{w}_{t}^{k} & +\left[-\tilde{L}_{t} v_{t}\left(x, y_{t}\right)+c_{t}\left(y_{t}\right) v_{t}\left(x, y_{t}\right)\right. \\
& \left.+\tilde{a}_{t}^{d+i, d+j}\left(y_{t}\right) v_{t y^{i} y^{j}}\left(x, y_{t}\right)+\Phi_{t}^{i k} \tilde{\beta}_{t}^{k} v_{t y^{i}}\left(x, y_{t}\right)\right] d t .
\end{aligned}
$$

We also rewrite (2.9) in terms of $\tilde{w}_{t}$ to get

$$
d \bar{\pi}_{t}(x)=\Lambda_{t}^{k}(x) \bar{\pi}_{t}(x) d \tilde{w}_{t}^{k}+\left[L_{t}^{*}(x) \bar{\pi}_{t}(x)+\Lambda_{t}^{k}(x) \bar{\pi}_{t}(x) \tilde{\beta}_{t}^{k}\right] d t .
$$

Then we use

$$
d\left[\bar{\pi}_{t}(x) v_{t}\left(x, y_{t}\right)\right]=v_{t}\left(x, y_{t}\right) d \bar{\pi}_{t}(x)+\bar{\pi}_{t}(x) d v_{t}\left(x, y_{t}\right)+d \bar{\pi}_{t}(x) d v_{t}\left(x, y_{t}\right),
$$

which after evaluating all terms and integrating over $x \in \mathbb{R}^{d}$ yields

$$
\begin{aligned}
& d\left(\bar{\pi}_{t}, v_{t}\left(\cdot, y_{t}\right)\right)=\left[\left(\Lambda_{t}^{k} \bar{\pi}_{t}, v_{t}\left(\cdot, y_{t}\right)\right)+\left(\bar{\pi}_{t}, v_{t y^{i}}\left(\cdot, y_{t}\right)\right) \Phi_{t}^{i k}\right] d \tilde{w}_{t}^{k} \\
& +\left[\left(\bar{\pi}_{t},-\tilde{L}_{t} v_{t}\left(\cdot, y_{t}\right)+c_{t}\left(y_{t}\right) v_{t}\left(\cdot, y_{t}\right)+\tilde{a}_{t}^{d+i, d+j}\left(y_{t}\right) v_{t y^{i} y^{j}}\left(\cdot, y_{t}\right)+\Phi_{t}^{i k} \tilde{\beta}_{t}^{k} v_{t y^{i}}\left(\cdot, y_{t}\right)\right)\right. \\
& \left.+\left(L_{t}^{*} \bar{\pi}_{t}+\tilde{\beta}_{t}^{k} \Lambda_{t}^{k} \bar{\pi}_{t}, v_{t}\left(\cdot, y_{t}\right)\right)+\left(v_{t y^{i}}\left(\cdot, y_{t}\right) \Phi_{t}^{i k}, \Lambda_{t}^{k} \bar{\pi}_{t}\right)\right] d t .
\end{aligned}
$$

Next we integrate by parts observing that

$$
\begin{gathered}
a_{t}^{i j}(x)=\tilde{a}_{t}^{i j}\left(x, y_{t}\right), \quad \tilde{b}_{t}^{i}\left(x, y_{t}\right)=b_{t}^{i}(x) \quad i, j \leq d, \\
2 \tilde{a}_{t}^{i, d+j}\left(x, y_{t}\right)=\left(\theta\left(t, x, y_{t}\right) \Theta_{t}^{*}\right)^{i j}=\sigma_{t}^{i k}(x) \Phi_{t}^{k j} \quad i \leq d, j \leq d_{1}-d, \\
a_{t}^{i j}(x) v_{t x^{i} x^{j}}\left(x, y_{t}\right)+\tilde{a}_{t}^{d+i, d+j}\left(y_{t}\right) v_{t y^{i} y^{j}}\left(x, y_{t}\right)+b_{t}^{i}(x) v_{t x^{i}}\left(x, y_{t}\right) \\
=\tilde{L}_{t} v_{t}\left(x, y_{t}\right)-\sigma_{t}^{i k}(x) \Phi_{t}^{k j} v_{t x^{i} y^{j}}\left(x, y_{t}\right)-\tilde{b}_{t}^{d+i}\left(x, y_{t}\right) v_{t y^{i}}\left(x, y_{t}\right) \\
=\tilde{L}_{t} v_{t}\left(x, y_{t}\right)-\sigma_{t}^{i k}(x) \Phi_{t}^{k j} v_{t x^{i} y^{j}}\left(x, y_{t}\right)-\Phi_{t}^{i k} \beta_{t}^{k}(x) v_{t y^{i}}\left(x, y_{t}\right)
\end{gathered}
$$

and obtain

$$
\begin{gathered}
\left(L_{t}^{*} \bar{\pi}_{t}, v_{t}\left(\cdot, y_{t}\right)\right)=\left(\bar{\pi}_{t}, L_{t} v_{t}\left(\cdot, y_{t}\right)\right)=\left(\bar{\pi}_{t}, a_{t}^{i j} v_{t x^{i} x^{j}}\left(\cdot, y_{t}\right)\right)+\left(\bar{\pi}_{t}, b_{t}^{i} v_{t x^{i}}\left(\cdot, y_{t}\right)\right) \\
\left(L_{t}^{*} \bar{\pi}_{t}, v_{t}\left(\cdot, y_{t}\right)\right)+\left(\bar{\pi}_{t},-\tilde{L}_{t} v_{t}\left(\cdot, y_{t}\right)+\tilde{a}_{t}^{d+i, d+j}\left(y_{t}\right) v_{t y^{i} y^{j}}\left(\cdot, y_{t}\right)\right) \\
=-\Phi_{t}^{k j}\left(\bar{\pi}_{t}, \sigma_{t}^{i k} v_{t x^{i} y^{j}}\left(\cdot, y_{t}\right)\right)-\Phi_{t}^{i k}\left(\bar{\pi}_{t}, \beta_{t}^{k} v_{t y^{i}}\left(\cdot, y_{t}\right)\right)
\end{gathered}
$$

Then (3.11) becomes

$$
\begin{aligned}
& d\left(\bar{\pi}_{t}, v_{t}\left(\cdot, y_{t}\right)\right)=\left[\left(\Lambda_{t}^{k} \bar{\pi}_{t}, v_{t}\left(\cdot, y_{t}\right)\right)+\left(\bar{\pi}_{t}, v_{t y^{i}}\left(\cdot, y_{t}\right)\right) \Phi_{t}^{i k}\right] d \tilde{w}_{t}^{k} \\
& \quad+\left[c_{t}\left(y_{t}\right)\left(\bar{\pi}_{t}, v_{t}\left(\cdot, y_{t}\right)\right)-\Phi_{t}^{k j}\left(\bar{\pi}_{t}, \sigma_{t}^{i k} v_{t x^{i} y^{j}}\left(\cdot, y_{t}\right)\right)-\Phi_{t}^{i k}\left(\bar{\pi}_{t}, \beta_{t}^{k} v_{t y^{i}}\left(\cdot, y_{t}\right)\right)\right. \\
& \left.\quad+\Phi_{t}^{i k} \tilde{\beta}_{t}^{k}\left(\bar{\pi}_{t}, v_{t y^{i}}\left(\cdot, y_{t}\right)\right)+\tilde{\beta}_{t}^{k}\left(\Lambda_{t}^{k} \bar{\pi}_{t}, v_{t}\left(\cdot, y_{t}\right)\right)+\Phi_{t}^{i k}\left(v_{t y^{i}}\left(\cdot, y_{t}\right), \Lambda_{t}^{k} \bar{\pi}_{t}\right)\right] d t .
\end{aligned}
$$


By using

$$
\begin{gathered}
-\Phi_{t}^{k j}\left(\bar{\pi}_{t}, \sigma_{t}^{i k} v_{t x^{i} y^{j}}\left(\cdot, y_{t}\right)\right)=\Phi_{t}^{k j}\left(\left(\sigma_{t}^{i k} \bar{\pi}_{t}\right)_{x^{i}}, v_{t y^{j}}\left(\cdot, y_{t}\right)\right) \\
=-\Phi_{t}^{i k}\left(\Lambda_{t}^{k} \bar{\pi}_{t}, v_{t y^{i}}\left(\cdot, y_{t}\right)\right)+\Phi_{t}^{i k}\left(\beta_{t}^{k} \bar{\pi}_{t}, v_{t y^{i}}\left(\cdot, y_{t}\right)\right),
\end{gathered}
$$

we further reduce (3.11) to

$$
\begin{aligned}
d\left(\bar{\pi}_{t}, v_{t}\left(\cdot, y_{t}\right)\right)=\left[\left(\Lambda_{t}^{k} \bar{\pi}_{t}, v_{t}\left(\cdot, y_{t}\right)\right)+\left(\bar{\pi}_{t}, v_{t y^{i}}\left(\cdot, y_{t}\right)\right)\right. & \left.\Phi_{t}^{i k}\right]\left(d \tilde{w}_{t}^{k}+\tilde{\beta}_{t}^{k} d t\right) \\
& +c_{t}\left(y_{t}\right)\left(\bar{\pi}_{t}, v_{t}\left(\cdot, y_{t}\right)\right) d t .
\end{aligned}
$$

Finally, for $\xi_{t}$ defined in (3.9), by Itô's formula we get

$$
\begin{gathered}
d \xi_{t}=\rho_{t} e^{-\int_{0}^{t} c_{s}\left(y_{s}\right) d s}\left[\left(\Lambda_{t}^{k} \bar{\pi}_{t}, v_{t}\left(\cdot, y_{t}\right)\right)\right. \\
\left.+\left(\bar{\pi}_{t}, v_{t y^{i}}\left(\cdot, y_{t}\right)\right) \Phi_{t}^{i k}-\tilde{\beta}_{t}^{k}\left(\bar{\pi}_{t}, v_{t}\left(\cdot, y_{t}\right)\right)\right] d \tilde{w}_{t}^{k},
\end{gathered}
$$

which shows that $\xi_{t}$ is at least a local martingale.

Remark 3.1. For $t \leq T$, define

$$
S_{t, T} \varphi(z)=v_{t}(z), \quad Q_{t, T}^{k} \varphi(z)=\beta_{t}(x) v_{t}(z)+\sigma_{t}^{i k}(x) v_{t x^{i}}(z)+\Phi_{t}^{i k} v_{t y^{i}}(z),
$$

where in the definition of $v$ we take $c \equiv 0$. Then (3.13) implies that

$$
\left(\bar{\pi}_{T}, \varphi\left(\cdot, y_{T}\right)\right)=\left(\pi_{0}, S_{0, T} \varphi\left(\cdot, y_{0}\right)\right)+\int_{0}^{T}\left(\bar{\pi}_{s}, Q_{s, T}^{k} \varphi\left(\cdot, y_{s}\right)\right)\left(d \tilde{w}_{s}^{k}+\tilde{\beta}_{s}^{k} d s\right)
$$

and generally for any $t \leq T$

$$
\left(\bar{\pi}_{t}, \varphi\left(\cdot, y_{t}\right)\right)=\left(\pi_{0}, S_{0, t} \varphi\left(\cdot, y_{0}\right)\right)+\int_{0}^{t}\left(\bar{\pi}_{s}, Q_{s, t}^{k} \varphi\left(\cdot, y_{s}\right)\right)\left(d \tilde{w}_{s}^{k}+\tilde{\beta}_{s}^{k} d s\right) .
$$

Following an idea in [6], one can iterate the last formula to get, for $d \hat{w}_{s}:=$ $d \tilde{w}_{s}^{k}+\tilde{\beta}_{s}^{k} d s$,

$$
\begin{aligned}
\left(\bar{\pi}_{t}, \varphi\left(\cdot, y_{t}\right)\right) & =\left(\pi_{0}, S_{0, t} \varphi\left(\cdot, y_{0}\right)\right)+\int_{0}^{t}\left(\pi_{0}, S_{0, s_{1}} Q_{s_{1}, t}^{k_{1}} \varphi\left(\cdot, y_{0}\right)\right) d \hat{w}_{s_{1}}^{k_{1}} \\
+ & \int_{0}^{t} \int_{0}^{s_{1}}\left(\bar{\pi}_{s_{2}}, Q_{s_{2}, s_{1}}^{k_{2}} Q_{s_{1}, t}^{k_{1}} \varphi\left(\cdot, y_{s_{2}}\right)\right) d \hat{w}_{s_{2}}^{k_{2}} d \hat{w}_{s_{1}}^{k_{1}}=\left(\pi_{0}, S_{0, t} \varphi\left(\cdot, y_{0}\right)\right) \\
+ & \sum_{i=1}^{n} \int_{0}^{t} \int_{0}^{s_{1}} \ldots \int_{0}^{s_{i-1}}\left(\pi_{0}, S_{0, s_{i}} Q_{s_{i}, s_{i-1}}^{k_{i}} \ldots Q_{s_{1}, t}^{k_{1}} \varphi\left(\cdot, y_{0}\right)\right) d \hat{w}_{s_{i}}^{k_{i}} \ldots d \hat{w}_{s_{1}}^{k_{1}} \\
& +\int_{0}^{t} \int_{0}^{s_{1}} \ldots \int_{0}^{s_{n}}\left(\pi_{0}, Q_{s_{n+1}, s_{n}}^{k_{n+1}} \ldots Q_{s_{1}, t}^{k_{1}} \varphi\left(\cdot, y_{0}\right)\right) d \hat{w}_{s_{n+1}}^{k_{n+1} \ldots d \hat{w}_{s_{1}}^{k_{1}} .}
\end{aligned}
$$

By using Girsanov's theorem, one can show that, on the probability space $(\Omega, \mathcal{F}, \tilde{P})$ with $\tilde{P}(d \omega)=\rho_{T}^{-1} P(d \omega)$ ), the process $\hat{w}_{t}$ is a Wiener process (see the proof of Lemma 5.1). Therefore, multiple stochastic integrals in (3.15) are orthogonal and if in the sum we replace $n$ with $\infty$, the series converges. One can prove that the last term goes to zero as $n \rightarrow \infty$ (cf. [6]) and then one obtains an "explicit" formula for $\left(\bar{\pi}_{t}, \varphi\left(\cdot, y_{t}\right)\right)$ in terms of multiple stochastic integrals of functions, whose dependence on $\omega$ is only through $y_{0}$, with respect to $d \hat{w}_{s}=\Psi_{s} d y_{s}$ the latter being uniquely determined by the observation process $y$. 


\section{SPDES IN THE SPACES $\mathcal{H}_{p}^{n}(T)$}

In this section we recall some definitions and results from [5] or [4]. In order not to introduce new letters, in this sections we use notation $a^{i j}, b^{i}, \sigma^{i k}$ for objects sometimes different from those in Sections 2 and 3. Fix a number $p \in[2, \infty)$.

Let $\mathcal{D}$ be the space of real-valued Schwarz distributions defined on $C_{0}^{\infty}\left(\mathbb{R}^{d}\right)$. By $H_{p}^{\gamma}=H_{p}^{\gamma}\left(\mathbb{R}^{d}\right)$ we denote the Banach space of Bessel potentials as stated before Assumption 2.6 with norm $\|\cdot\|_{\gamma, p}$ and with $\|\cdot\|_{p}=\|\cdot\|_{0, p}$. Recall (see, for instance [14]) that for integers $n \geq 0$ the space $H_{p}^{n}$ coincides with the Sobolev space $W_{p}^{n}=W_{p}^{n}\left(\mathbb{R}^{d}\right)$. We allow elements of $H_{p}^{\gamma}$ to be vector- or matrix-valued functions. Next, denote

$$
\mathbb{H}_{p}^{\gamma}(T)=L_{p}\left(\Omega \times(0, T], \mathcal{P}, H_{p}^{\gamma}\right), \quad \mathbb{L}_{\ldots \ldots}=\mathbb{H}_{\ldots \ldots}^{0} \ldots
$$

The norms in these spaces are defined in an obvious way. As it is conventional, elements of spaces $\mathbb{H}_{p}^{\gamma}(T)$ are treated as functions rather than distributions or classes of equivalent functions, and if we know that a function of this class has a modification with better properties, then we always consider this modification. For instance, if we take $u \in H_{p}^{\gamma}$ and $\gamma-d / p>0$, then $u$ has a bounded continuous modification, but we talk about $\sup _{x} u(x)$ instead of sup of this modification. Also elements of spaces $\mathbb{H}_{p}^{\gamma}(T)$ need not be defined or belong to $H_{p}^{\gamma}$ for all $(\omega, t) \in \Omega \times(0, T]$. As usual, these properties are needed only for almost all $(\omega, t)$.

Definition 4.1. For a $\mathcal{D}$-valued function $u_{t}(x)=u_{t}(\omega, x)$ such that $u \in \mathbb{H}_{p}^{\gamma}(T)$, we write $u \in \mathcal{H}_{p}^{\gamma}(T)$ if $u_{t x x} \in \mathbb{H}_{p}^{\gamma-2}(T)$ and there exist a real-valued $f_{t} \in \mathbb{H}_{p}^{\gamma-2}(T)$ and $\mathbb{R}^{d_{2}}$-valued $g_{t} \in \mathbb{H}_{p}^{\gamma-1}(T)$ such that for any $\phi \in C_{0}^{\infty}\left(\mathbb{R}^{d}\right)$, with probability 1 the equality

$$
\left(u_{t}, \phi\right)=\left(u_{0}, \phi\right)+\int_{0}^{t}\left(f_{s}, \phi\right) d s+\int_{0}^{t}\left(g_{s}^{k}, \phi\right) d w_{s}^{k}
$$

holds for all $t \leq T$ and $u_{0} \in L_{p}\left(\Omega, \mathcal{F}_{0}, H_{p}^{\gamma-2 / p}\right)$. In that case we also define

$$
\|u\|_{\mathcal{H}_{p}^{\gamma}(T)}^{p}=\left\|u_{x x}\right\|_{\mathbb{H}_{p}^{\gamma-2}(T)}^{p}+\|f\|_{\mathbb{H}_{p}^{\gamma-2}(T)}^{p}+\|g\|_{\mathbb{H}_{p}^{\gamma}(T)}^{p}+E\left\|u_{0}\right\|_{\gamma-2 / p, p}^{p}
$$

and write

$$
d u_{t}=f_{t} d t+g_{t}^{k} d w_{t}^{k} \quad t \leq T .
$$

Notice a useful property of spaces $\mathcal{H}_{p}^{\gamma}(T)$ proved, for instance, in Section 7 of [5].

Theorem 4.2. If $u \in \mathcal{H}_{p}^{\gamma}(T)$, then $u \in C\left([0, T], H_{p}^{\gamma-1}\right)$ (a.s.).

We will also use a version of Itô's formula.

Lemma 4.3. Let $\phi_{t}(x)=\phi_{t}(\omega, x), \xi_{t}(x)=\xi_{t}(\omega, x)$, and $\eta_{t}^{k}(x)=\eta_{t}^{k}(\omega, x), k=$ $1, \ldots, d_{2}$, be real-valued functions on $\Omega \times[0, T] \times \mathbb{R}^{d}$ such that

(i) the functions $\phi_{t}, \xi_{t}$, and $\eta_{t}^{k}$ are continuous with respect to $x$ for any $(\omega, t)$ and there are constants $N<\infty$ and $\varepsilon>0$ such that

$$
\left|\phi_{t}(x)\right|+\left|\xi_{t}(x)\right|+\left|\eta_{t}^{k}(x)\right| \leq N e^{-\varepsilon|x|}, \quad \forall \omega, t, x, k ;
$$

(ii) for each $x \in \mathbb{R}^{d}$, the processes $\phi_{t}, \xi_{t}$, and $\eta_{t}$ are $\mathcal{F}_{t}$-adapted and

$$
d \phi_{t}(x)=\xi_{t}(x) d t+\eta_{t}^{k}(x) d w_{t}^{k}
$$

Then for any $u \in \mathcal{H}_{p}^{2}(T)$ with $d u_{t}=f_{t} d t+g_{t}^{k} d w_{t}^{k}$ we have

$$
d\left(u_{t}, \phi_{t}\right)=\left[\left(f_{t}, \phi_{t}\right)+\left(u_{t}, \xi_{t}\right)+\left(g_{t}^{k}, \eta_{t}^{k}\right)\right] d t+d m_{t},
$$


where $m_{t}$ is a square integrable martingale on $[0, T]$ defined by

$$
m_{t}=\int_{0}^{t}\left[\left(g_{s}^{k}, \phi_{s}\right)+\left(u_{s}, \eta_{s}^{k}\right)\right] d w_{s}^{k}
$$

Proof. Take a nonnegative function $\zeta \in C_{0}^{\infty}\left(\mathbb{R}^{d}\right)$ with unit integral and for $n \geq 1$ define $\zeta_{n}(x)=n^{d} \zeta(n x), h_{t}^{(n)}(x)=\zeta_{n} * h_{t}(x)$. Then by definition, for any $x \in \mathbb{R}^{d}$

$$
d u_{t}^{(n)}(x)=f_{t}^{(n)}(x) d t+g_{t}^{(n) k}(x) d w_{t}^{k} .
$$

By Itô's formula

$$
\begin{aligned}
d\left[u_{t}^{(n)}(x) \phi_{t}(x)\right]=\left[f_{t}^{(n)}(x) \phi_{t}(x)+u_{t}^{(n)}(x) \xi_{t}(x)+g_{t}^{(n) k}(x) \eta_{t}^{k}(x)\right] d t \\
+\left[g_{t}^{(n) k}(x) \phi_{t}(x)+u_{t}^{(n)}(x) \eta_{t}^{k}(x)\right] d w_{t}^{k} .
\end{aligned}
$$

Here all functions are continuous in $x$ and by Hölder's inequality

$$
\begin{gathered}
E \int_{0}^{T}\left(\int_{\mathbb{R}^{d}}\left|g_{t}^{(n) k}(x) \phi_{t}(x)+u_{t}^{(n)}(x) \eta_{t}^{k}(x)\right| d x\right)^{2} d t \\
\leq N E \int_{0}^{T}\left(\int_{\mathbb{R}^{d}}\left[\left|g_{t}^{(n) k}(x)\right|^{p}+\left|u_{t}^{(n)}(x)\right|^{p}\right] d x\right)^{2 / p} d t \\
\leq N\left(\left\|g^{(n) k}\right\|_{\mathbb{L}_{p}(T)}^{2}+\left\|u^{(n)}\right\|_{\mathbb{L}_{p}(T)}^{2} \leq N\left(\left\|g^{k}\right\|_{\mathbb{L}_{p}(T)}^{2}+\|u\|_{\mathbb{L}_{p}(T)}^{2}\right)<\infty .\right.
\end{gathered}
$$

Similar inequality holds for the deterministic part in (4.4). Therefore (see, for instance, [11]), the stochastic Fubini's theorem is applicable, so that

$$
\begin{aligned}
d\left(u_{t}^{(n)}, \phi_{t}\right)=\left[\left(f_{t}^{(n)}, \phi_{t}\right)+\left(u_{t}^{(n)}, \xi_{t}\right)+\left(g_{t}^{(n) k}, \eta_{t}^{k}\right)\right] d t & \\
& +\left[\left(g_{t}^{(n) k}, \phi_{t}\right)+\left(u_{t}^{(n)}, \eta_{t}^{k}\right)\right] d w_{t}^{k} .
\end{aligned}
$$

Now we let $n \rightarrow \infty$. Notice that (a.s.)

$$
\sup _{t \leq T}\left|\left(u_{t}^{(n)}, \phi_{t}\right)-\left(u_{t}, \phi_{t}\right)\right| \leq N \sup _{t \leq T}\left\|u_{t}^{(n)}-u_{t}\right\|_{p} \rightarrow 0
$$

because of the continuity of $u_{t}$ in $L_{p}\left(\mathbb{R}^{d}\right)$ and well-known properties of mollifiers. Also

$$
\begin{aligned}
& E \int_{0}^{T}\left[\left(g_{t}^{(n) k}, \phi_{t}\right)+\left(u_{t}^{(n)}, \eta_{t}^{k}\right)-\left(g_{t}^{k}, \phi_{t}\right)+\left(u_{t}, \eta_{t}^{k}\right)\right]^{2} d t \\
& \quad \leq N E \int_{0}^{T}\left(\left\|g_{t}^{(n) k}-g_{t}^{k}\right\|_{p}^{2 / p}+\left\|u_{t}^{(n)}-u_{t}\right\|_{p}^{2 / p}\right) d t \\
& \quad \leq N\left(\left\|g^{(n) k}-g^{k}\right\|_{\mathbb{L}_{p}(T)}^{2}+\left\|u^{(n)}-u\right\|_{\mathbb{L}_{p}(T)}^{2}\right) \rightarrow 0 .
\end{aligned}
$$

In the same way one treats the deterministic part in (4.5) and one arrives at (4.3). The lemma is proved.

Consider the following equation

$$
d u_{t}=\left(a_{t}^{i j} u_{t x^{i} x^{j}}+b_{t}^{i} u_{t x^{i}}+c_{t} u_{t}+f_{t}\right) d t+\left(\sigma_{t}^{i k} u_{t x^{i}}+\nu_{t}^{k} u_{t}+g_{t}^{k}\right) d w_{t}^{k} \quad t \leq T,
$$

where (as always we use the summation convention and) $a^{i j}, b^{i}, c, f, \sigma^{i k}, \nu^{k}, g^{k}$ are some functions of $(\omega, t, x)$ specified below. Equation (4.6) will be understood in the sense of Definition 4.1. 
Fix $\gamma \in(-\infty, \infty)$ and fix a number $\kappa \in[0,1)$ such that $\kappa=0$ if $\gamma=0, \pm 1, \pm 2, \ldots$; otherwise $\kappa>0$ and is such that $|\gamma|+\kappa$ is not an integer. Define

$$
B^{|\gamma|+\kappa}= \begin{cases}B\left(\mathbb{R}^{d}\right) & \text { if } \gamma=0 \\ C^{|\gamma|-1,1}\left(\mathbb{R}^{d}\right) & \text { if } \gamma= \pm 1, \pm 2, \ldots \\ C^{|\gamma|+\kappa}\left(\mathbb{R}^{d}\right) & \text { otherwise }\end{cases}
$$

where $B\left(\mathbb{R}^{d}\right)$ is the Banach space of bounded functions on $\mathbb{R}^{d}, C^{|\gamma|-1,1}\left(\mathbb{R}^{d}\right)$ is the Banach space of $|\gamma|-1$ times continuously differentiable functions whose derivatives of $(|\gamma|-1)$ st order satisfy the Lipschitz condition on $\mathbb{R}^{d}, C^{|\gamma|+\kappa}\left(\mathbb{R}^{d}\right)$ is the usual Hölder space.

Next, take an $\varepsilon_{0} \in(0,1)$ and define

$$
\begin{aligned}
& \gamma_{b}=\gamma+\kappa \quad \text { if } \quad \gamma \geq 0, \quad \gamma_{b}=0 \quad \text { if } \quad \gamma \in(-1,0], \\
& \gamma_{\nu}=\gamma+\kappa+1 \quad \text { if } \quad \gamma \geq-1, \quad \gamma_{\nu}=0 \quad \text { if } \quad \gamma \in(-2,-1] \text {, } \\
& \gamma_{c}=\gamma+\kappa \quad \text { if } \quad \gamma \geq 0, \quad \gamma_{c}=0 \quad \text { if } \quad \gamma \in(-2,0] \text {, } \\
& \gamma_{b}=-\gamma-1+\varepsilon_{0} \quad \text { if } \quad \gamma \leq-1 \text {, } \\
& \gamma_{\nu}=-\gamma-2+\varepsilon_{0} \quad \text { if } \quad \gamma \leq-2 \text {, } \\
& \gamma_{c}=-\gamma-2+\varepsilon_{0} \quad \text { if } \quad \gamma \leq-2 \text {. }
\end{aligned}
$$

Assumption 4.4 (coercivity). For any $\omega \in \Omega, t \in[0, T], x, \lambda \in \mathbb{R}^{d}$, we have

$$
K|\lambda|^{2} \geq\left[a_{t}^{i j}(x)-\sigma_{t}^{i k} \sigma_{t}^{j k}(x)\right] \lambda^{i} \lambda^{j} \geq \delta|\lambda|^{2} .
$$

Assumption 4.5 (uniform continuity of $a$ and $\sigma$ ). For any $\varepsilon>0, i, j, k$, there exists a $\delta_{\varepsilon}>0$ such that

$$
\left|a_{t}^{i j}(x)-a_{t}^{i j}(y)\right|+\left|\sigma_{t}^{i k}(x)-\sigma_{t}^{i k}(y)\right| \leq \varepsilon
$$

whenever $|x-y| \leq \delta_{\varepsilon}, t \in[0, T], \omega \in \Omega$.

This assumption is actually used only if $\gamma=0$. And even then we need a stronger condition on $\sigma$. For other values of $\gamma$ we impose stronger conditions on $a$ and $\sigma$.

Assumption 4.6. For any $i, j, k$, the functions $a_{t}^{i j}(x), b_{t}^{i}(x), c_{t}(x), \sigma_{t}^{i k}(x)$, and $\nu_{t}^{k}(x)$ are real-valued $\mathcal{P} \times \mathcal{B}\left(\mathbb{R}^{d}\right)$-measurable functions, and for any $\omega \in \Omega$ and $t \in[0, T]$, we have

$$
a_{t}^{i j} \in B^{|\gamma|+\kappa}, \quad \sigma_{t}^{i k} \in B^{|\gamma+1|+\kappa}, \quad b_{t}^{i} \in B^{\gamma_{b}}, \quad c_{t} \in B^{\gamma_{c}}, \quad \nu_{t}^{k} \in B^{\gamma_{\nu}} .
$$

Observe that if $\gamma=0,(4.8)$ means that

$$
a_{t}^{i j}, b_{t}^{i}, c_{t} \in B\left(\mathbb{R}^{d}\right), \quad \sigma_{t}^{i k}, \nu_{t}^{k} \in C^{0,1}\left(\mathbb{R}^{d}\right),
$$

whereas if $\gamma=1,(4.8)$ means that

$$
a_{t}^{i j}, b_{t}^{i}, c_{t} \in C^{0,1}\left(\mathbb{R}^{d}\right), \quad \sigma_{t}^{i k}, \nu_{t}^{k} \in C^{1,1}\left(\mathbb{R}^{d}\right),
$$

Assumption 4.7. The functions $f_{t}$ and $g_{t}$ are predictable as functions taking values in $H_{p}^{\gamma}$ and $H_{p}^{\gamma+1}$, respectively.

Assumption 4.8. For any $t \in[0, T], \omega, i, j, k$,

$$
\left\|a_{t}^{i j}\right\|_{B|\gamma|+\kappa}+\left\|\sigma_{t}^{i k}\right\|_{B|\gamma+1|+\kappa}+\left\|b_{t}^{i}\right\|_{B^{\gamma_{b}}}+\left\|c_{t}\right\|_{B^{\gamma_{c}}}+\left\|\nu_{t}\right\|_{B^{\gamma_{\nu}}} \leq K
$$

and $f \in \mathbb{H}_{p}^{\gamma}(T), g \in \mathbb{H}_{p}^{\gamma+1}(T)$.

The following theorems are proved, for instance, in Section 5 of [5]. 
Theorem 4.9. Let Assumptions 4.4-4.8 be satisfied and let

$$
u_{0} \in L_{p}\left(\Omega, H_{p}^{\gamma+2-2 / p}\right) .
$$

Then the Cauchy problem for equation (4.6) on $[0, T]$ with the initial condition $u_{0}$ has a unique solution $u \in \mathcal{H}_{p}^{\gamma+2}(T)$. For this solution, we have

$$
\|u\|_{\mathcal{H}_{p}^{\gamma+2}(T)} \leq N\left\{\|f\|_{\mathbb{H}_{p}^{\gamma}(T)}+\|g\|_{\mathbb{H}_{p}^{\gamma+1}(T)}+\left(E\left\|u_{0}\right\|_{\gamma+2-2 / p, p}^{p}\right)^{1 / p}\right\}
$$

where the constant $N$ depends only on $d, d_{2}, \gamma, \kappa, \varepsilon_{0}, p, \delta, K, T$, and the function $\delta_{\varepsilon}$.

Theorem 4.10. Let the assumptions of Theorem 4.9 be satisfied and let $u_{0} \geq$ $0, f \geq 0$, and $g \equiv 0$. Then $u_{t} \geq 0$ for all $t \in[0, T]$ (a.s.).

Proof of Lemma 2.9. First notice that problem (2.9) has the form (4.6) with $f \equiv g \equiv 0$, which is seen if we perform all differentiations in the definitions of $L^{*}$ and $\Lambda$ and notice that Lipschitz continuous functions have bounded derivatives. After this it is also quite easy to see that all the assumptions of Theorem 4.9 are satisfied with $\gamma=0$.

Therefore the unique solvability of $(2.9)$ in $\mathcal{H}_{p}^{2}(T)$ follows from Theorem 4.9 and the fact that $\bar{\pi} \geq 0$ follows from Theorem 4.10. The lemma is proved.

We also need some facts concerning the deterministic problem (3.3). Unfortunately one cannot get them from general results in [8] or [13] just because our coefficients are not supposed to have any regularity in $t$. In order to be able to apply Theorem 4.9 (with $\sigma \equiv \nu \equiv g \equiv 0$ ) to (3.3) we notice that, for the function $u_{t}(x):=v_{T-t}(x)$, problem (3.3) takes the form

$$
\begin{aligned}
d u_{t}(z) & =\left(\tilde{L}_{T-t}(z) u_{t}(z)-c_{T-t}(y) u_{t}(z)\right) d t, \quad t \in[0, T], z \in \mathbb{R}^{d_{1}}, \\
u_{0}(z) & =\varphi(z), \quad z \in \mathbb{R}^{d_{1}} .
\end{aligned}
$$

Lemma 4.11. Let $\varphi \in C_{0}^{\infty}\left(\mathbb{R}^{d_{1}}\right)$ and $c \in C^{1}\left([0, T] \times \mathbb{R}^{d_{1}-d}\right), c \geq 0$. Then there exists a unique function $v$ on $[0, T] \times \mathbb{R}^{d_{1}}$ possessing the following properties

(i) for any $t \in[0, T], q \in(1, \infty)$, and $\varepsilon \in(0,1)$, the function $v_{t}$ is continuous as a $C^{2+\varepsilon}\left(\mathbb{R}^{d_{1}}\right)$-valued function on $[0, T]$;

(ii) for any $t \in[0, T]$ and $z \in \mathbb{R}^{d_{1}}$, it holds that

$$
v_{t}(z)=\varphi(z)+\int_{t}^{T}\left[\tilde{L}_{s}(z) v_{s}(z)-c_{s}(y) v_{s}(z)\right] d s .
$$

(iii) There exist constants $N<\infty, \varepsilon>0$ such that $\left|v_{t}(z)\right|+\left|v_{t z}(z)\right|+\left|v_{t z z}(z)\right| \leq$ $N \exp (-\varepsilon|z|)$ for all $t \in[0, T]$ and $z \in \mathbb{R}^{d_{1}}$.

Proof. It suffices to prove analogous fact for (4.9). Observe that the assumptions of Theorem 4.9 are satisfied with $\gamma=1$ and $q$ in place of $p$ for any $q \geq 2$. Therefore, for any $q \geq 2$ in $\mathcal{H}_{q}^{3}(T)$, there exists a unique solution $u$ of (4.9). By Corollary 5.11 of [5], this solution is the same for all $q \geq 2$, so that $u \in \mathcal{H}_{q}^{3}(T)$ for all $q \geq 2$. We have applied the results of [5] to deterministic case in which there is no need to consider random functions and one can confine oneself to smaller spaces.

Let $\mathfrak{H}_{q}^{\gamma}(T)$ be the space of all deterministic functions belonging to $\mathcal{H}_{q}^{\gamma}(T)$, that is the space of all measurable $H_{q}^{\gamma}\left(\mathbb{R}^{d_{1}}\right)$-valued function $u_{t}$ defined on $t \in[0, T]$ and such that, for a measurable $H_{q}^{\gamma-2}\left(\mathbb{R}^{d_{1}}\right)$-valued function $f_{t}$ and all $t \in[0,1]$ and $\psi \in C_{0}^{\infty}\left(\mathbb{R}^{d_{1}}\right)$, we have

$$
\left(u_{t}, \psi\right)=\left(u_{0}, \psi\right)+\int_{0}^{t}\left(f_{s}, \psi\right) d s
$$


and

$$
\int_{0}^{T}\left\{\left\|u_{t x x}\right\|_{H_{p}^{\gamma-2}\left(\mathbb{R}^{\left.d_{1}\right)}\right.}^{q}+\left\|f_{t}\right\|_{H_{p}^{\gamma-2}\left(\mathbb{R}^{d_{1}}\right)}^{q}\right\} d t<\infty .
$$

By throwing away all stochastic integrals in [5] one easily shows that the function $u$ constructed above is actually deterministic, so that $u \in \mathfrak{H}_{q}^{3}(T)$ for all $q \geq 2$. As a matter of fact, one can see that in the deterministic case the restriction $q \geq 2$ is never used and $u \in \mathfrak{H}_{q}^{3}(T)$ for all $q>1$. However, we will not use the last observation.

Now we use embedding Theorem 7.2 of [5] which is quite formally available for $\mathfrak{H}_{p}^{\gamma}(T)$ since $\mathfrak{H}_{p}^{\gamma}(T) \subset \mathcal{H}_{p}^{\gamma}(T)$. Then we get that, for $q>2$ and $1 / 2>\varepsilon>1 / q$,

$$
\left\|u_{t}-u_{s}\right\|_{3-2 \varepsilon, q} \leq N|t-s|^{\varepsilon-1 / q} \quad \forall s, t \in[0, T],
$$

where $N$ is independent of $s, t$. By remembering the Sobolev embedding theorem: $H_{q}^{\gamma}\left(\mathbb{R}^{d_{1}}\right) \subset C^{\gamma-d_{1} / q}$ if $\gamma-d_{1} / q>0$ and $\gamma-d_{1} / q$ is not an integer, and choosing $q$ large and $\varepsilon$ small, we get that (4.10) implies the continuity of $u_{t}$ in $C^{2+\varepsilon}\left(\mathbb{R}^{d_{1}}\right)$.

Next, we notice that, by definition, (4.9) means that, for any $\psi \in C_{0}^{\infty}\left(\mathbb{R}^{d_{1}}\right)$ and $t \in[0, T]$,

$$
\left(u_{t}, \psi\right)=(\varphi, \psi)+\int_{0}^{t}\left(\tilde{L}_{T-s} u_{s}-c_{T-s} u_{s}, \psi\right) d s
$$

Since here all functions involved are continuous and bounded in $z$, we have

$$
\left(u_{t}-\varphi-\int_{0}^{t}\left(\tilde{L}_{T-s} u_{s}-c_{T-s} u_{s}\right) d s, \psi\right)=0,
$$

which in turn implies that

$$
u_{t}(z)=\varphi(z)+\int_{0}^{t}\left(\tilde{L}_{T-s}(z) u_{s}(z)-c_{T-s}(z) u_{s}(z)\right) d s
$$

for all $t \in[0, T]$ and $z \in \mathbb{R}^{d_{1}}$.

To prove the last assertion of the lemma, observe that $v$ belongs to the space of functions whose first derivative in time and second order derivatives in $z$ are summable (actually bounded) to the power $d_{1}+1$ over any bounded region in $[0, T] \times \mathbb{R}^{d_{1}}$. In addition $z_{t}$ is a uniformly nondegenerate process. Therefore (see Theorem 2.10.1 of [2]), Itô's formula is applicable to $v_{t}\left(z_{t}(s, z)\right)$, where $z_{t}(s, z)$ is the unique solution of $(2.8)$ for $t \in[s, T]$ with initial value $z_{s}=z$. Therefore,

$$
\begin{gathered}
v_{s}(z)=E \varphi\left(z_{T}(s, z)\right) \exp \left(-\int_{s}^{T} c_{r}\left(z_{r}(s, z)\right) d r\right) \\
\left|v_{s}(z)\right| \leq \sup |\varphi| P\{\tau(s, z) \leq T\} \leq \sup |\varphi| e^{T} E I_{\tau(s, z)<\infty} e^{-\tau(s, z)},
\end{gathered}
$$

where $\tau(s, z)$ is the first time $z_{t}(s, z)$ hits $\{z:|z| \leq R\}$ and $R$ is such that $\varphi(z)=0$ for $|z| \geq R$. By Lemma 2.4.2 of [2], the last expectation is less than $N \exp (-\varepsilon|z|)$ with $N$ and $\varepsilon>0$ independent of $z$, which gives us part of the asserted estimates in (iii).

It turns out that the remaining part follows from this estimate and from the boundedness of norms of $v_{t z}$ and $v_{t z z}$ in $C^{1 / 2}\left(\mathbb{R}^{d_{1}}\right)$, which is asserted in (i). Indeed, for instance, if $\xi$ is a unit vector in $\mathbb{R}^{d_{1}}$, then, for any $h \in(0,1]$,

$$
\begin{gathered}
\left|v_{t z^{i} z^{j}}(z) \xi^{i} \xi^{j}\right| \leq\left|v_{t z^{i} z^{j}}(z) \xi^{i} \xi^{j}-h^{-2}\left[v_{t}(z+h \xi)-2 v_{t}(z)+v_{t}(z+h \xi)\right]\right| \\
+h^{-2}\left|v_{t}(z+h \xi)-2 v_{t}(z)+v_{t}(z+h)\right| \leq N h^{1 / 2}|| v_{t z z} \|_{C^{1 / 2}\left(\mathbb{R}^{\left.d_{1}\right)}\right.}+N h^{-2} e^{-\varepsilon|z|}
\end{gathered}
$$


with $N$ independent of $t, z$, and $\xi$. With $h=\exp (-\varepsilon|z| / 4)$, we have $\left|v_{t z^{i} z^{j}} \xi^{i} \xi^{j}\right| \leq$ $N \exp (-\varepsilon|z| / 8)$ and since this holds for any unit $\xi$, we also have the same estimate for $\left|v_{t z^{i} z^{j}}\right|$. The lemma is proved.

\section{Proof of Theorem 2.10}

For any $x$ the function $v_{t}(x, \cdot)$ from Lemma 4.11 belongs to the space of functions whose first derivative in time and second order derivatives in $y$ are summable (actually bounded) to the power $d_{1}-d+1$ over any bounded region in $[0, T] \times \mathbb{R}^{d_{1}}$. In addition $y_{t}$ is a uniformly nondegenerate process. Therefore (see Theorem 2.10.1 of [2]), Itô's formula is applicable to $v\left(t, x, y_{t}\right)$ and this proves (3.10)

After this, Lemma 4.3 yields (3.11). To justify integrating by parts, notice that, by definition, for any $u \in \mathcal{D}\left(\mathbb{R}^{d}\right)$ and $\phi \in C_{0}^{\infty}\left(\mathbb{R}^{d}\right)$ we have

$$
\left(u_{x^{i}}, \phi\right)=-\left(u, \phi_{x^{i}}\right), \quad\left(u_{x^{i} x^{j}}, \phi\right)=\left(u, \phi_{x^{i} x^{j}}\right) .
$$

If we know that the generalized derivatives of $u$ are usual functions summable to some power $p \geq 1$, which is the case for $u \in H_{p}^{2}\left(\mathbb{R}^{d}\right)$, these formulas extend easily to functions $\phi$ having continuous derivatives and decreasing along with derivatives sufficiently fast.

Due to this observation, the integration by parts following (3.11) is legitimate. Hence (3.14) holds indeed.

Next by using Doob's inequality and boundedness of $\tilde{\beta}$, we obtain

$$
\begin{aligned}
E \sup _{t \leq T} \rho_{t}^{2} \leq 4 \sup _{t \leq T} E \rho_{T}^{2}= & 4 E \exp \left(-2 \int_{0}^{T} \tilde{\beta}_{s} d \tilde{w}_{s}-\int_{0}^{t}\left|\tilde{\beta}_{T}\right|^{2} d s\right) \\
& \leq N_{1} E \exp \left(-2 \int_{0}^{T} \tilde{\beta}_{s} d \tilde{w}_{s}-2 \int_{0}^{t}\left|\tilde{\beta}_{T}\right|^{2} d s\right)=N_{1} .
\end{aligned}
$$

In addition, by Lemma 4.3,

$$
E \int_{0}^{T}\left[\left(\Lambda_{t}^{k} \bar{\pi}_{t}, v_{t}\left(\cdot, y_{t}\right)\right)+\left(\bar{\pi}_{t}, v_{t y^{i}}\left(\cdot, y_{t}\right)\right) \Phi_{t}^{i k}-\tilde{\beta}_{t}^{k}\left(\bar{\pi}_{t}, v_{t}\left(\cdot, y_{t}\right)\right)\right]^{2} d t<\infty .
$$

It follows easily that

$$
E\left(\int_{0}^{T} \rho_{t}^{2}\left[\left(\Lambda_{t}^{k} \bar{\pi}_{t}, v_{t}\left(\cdot, y_{t}\right)\right)+\left(\bar{\pi}_{t}, v_{t y^{i}}\left(\cdot, y_{t}\right)\right) \Phi_{t}^{i k}-\tilde{\beta}_{t}^{k}\left(\bar{\pi}_{t}, v_{t}\left(\cdot, y_{t}\right)\right)\right]^{2} d t\right)^{1 / 2}<\infty
$$

which proves that $\xi_{t}$ is a martingale.

In particular, (3.7) holds. We want to derive from here that

$$
E e^{-\int_{0}^{T} c_{s}\left(y_{s}\right) d s} \varphi\left(z_{T}\right)=E e^{-\int_{0}^{T} c_{s}\left(y_{s}\right) d s} \bar{\rho}_{T} \int_{\mathbb{R}^{d}} \varphi\left(x, y_{T}\right) \bar{\pi}_{T}(x) d x
$$

where $\bar{\rho}_{t}$ is taken from (3.1). The following lemma implies that (5.2) holds indeed.

Lemma 5.1. The function $\bar{\pi}_{t}$ is $\mathcal{F}_{t}^{y}$-adapted.

Proof. Define

$$
\hat{w}_{t}=\int_{0}^{t} \Psi_{t} d y_{t}
$$

Since $\Psi_{t}$ is $\mathcal{F}_{t}^{y}$-adapted, the process $\hat{w}_{t}$ is $\mathcal{F}_{t}^{y}$-adapted too. We want to change the probability measure so that $\hat{w}_{t}$ would become a Wiener process with respect to this 
new measure. Define

$$
Q(d \omega)=\rho_{T}(\omega) P(d \omega)=\exp \left(-\int_{0}^{T} \tilde{\beta}_{s} d \tilde{w}_{s}-\frac{1}{2} \int_{0}^{T}\left|\tilde{\beta}_{s}\right|^{2} d s\right)(\omega) P(d \omega) .
$$

The process $\rho_{t}$ is an exponential local martingale. Similarly to (5.1), one proves that it is square integrable, so that $Q$ is a probability measure. Since

$$
d \hat{w}_{t}=\tilde{\beta}_{t} d t+d \tilde{w}_{t}
$$

and $\tilde{w}_{t}$ is a Wiener process on $(\Omega, \mathcal{F}, P)$, by Girsanov's theorem, $\hat{w}_{t}$ is a Wiener process on $(\Omega, \mathcal{F}, Q)$ with respect to the filtration $\left\{\mathcal{F}_{t}\right\}$. As has been noticed before, it is $\mathcal{F}_{t}^{y}$-adapted and, obviously, $\mathcal{F}_{t}^{y} \subset \mathcal{F}_{t}$, so that $\left(\hat{w}_{t}, \mathcal{F}_{t}^{y}\right)$ is a Wiener process. Now rewrite (2.9) as

$$
d \bar{\pi}_{t}(x)=L_{t}^{*}(x) \bar{\pi}_{t}(x) d t+\Lambda_{t}^{k}(x) \bar{\pi}_{t}(x) d \hat{w}_{t}^{r},
$$

and consider this equation relative to $\left(\Omega, \mathcal{F}, \mathcal{F}_{t}^{y}, Q\right)$. By the theory in [5], this equation with initial data $\pi_{0}$ has a unique $\mathcal{F}_{t}^{y}$-adapted solution belonging to $\mathcal{H}_{p}^{2}(Q, T)$, where by $\mathcal{H}_{p}^{2}(Q, T)$ we mean the space $\mathcal{H}_{p}^{2}(T)$ constructed on the basis of the new probability measure $Q$.

We want to derive that $\bar{\pi}_{t}$ is $\mathcal{F}_{t}^{y}$-adapted from the uniqueness. The only obstacle is that the norms in $\mathcal{H}_{p}^{2}(Q, T)$ and $\mathcal{H}_{p}^{2}(P, T)$ are different. To overcome this obstacle, we are going to use stopping times.

If $\tau \leq T$ is an $\mathcal{F}_{t}$-stopping time, define $\mathbb{H}_{p}^{\gamma}(\tau)=L_{p}\left(\left(0, \tau \rrbracket, \mathcal{P}, H_{p}^{\gamma}\right)\right.$ and define $\mathcal{H}_{p}^{\gamma}(\tau)$ following Definition 4.1 by replacing $\mathbb{H}_{p}^{\gamma}(T)$ with $\mathbb{H}_{p}^{\gamma}(\tau)$. It turns out that Theorem 4.9 holds true for these new spaces (see Section 5 of [5]). To distinguish between spaces $\mathcal{H}_{p}^{\gamma}(\tau)$ relative to different filtrations and probability measures we write $\mathcal{H}_{p}^{\gamma}(\mathcal{F},, P, \tau)$ for the space $\mathcal{H}_{p}^{\gamma}(\tau)$ corresponding to probability measure $P$ and filtration $\left\{\mathcal{F}_{t}\right\}$.

Now let $\tilde{\pi}_{t}$ be the unique $\mathcal{H}_{p}^{2}\left(\mathcal{F}^{y}, Q, T\right)$-solution of (5.3) with initial data $\pi_{0}$. Write $d \tilde{\pi}_{t}=\tilde{f}_{t} d t+\tilde{g}_{t}^{k} d \hat{w}_{t}^{k}$ and for integers $n$ define

$$
\tau(n)=T \wedge \inf \left\{t \geq 0: \int_{0}^{t}\left[\left\|\tilde{\pi}_{s x x}\right\|_{p}^{p}+\left\|\tilde{f}_{s}\right\|_{p}^{p}+\left\|\tilde{g}_{s}\right\|_{1, p}^{p}\right] d s \geq n\right\} .
$$

Obviously, $\tau(n)$ are $\mathcal{F}_{t}^{y}$-stopping times and $\mathcal{F}_{t}$-stopping times. Furthermore,

$$
\begin{gathered}
\|\tilde{\pi}\|_{\mathcal{H}_{p}^{2}(\mathcal{F}, P, \tau(n))}^{p}=E \int_{0}^{\tau(n)}\left[\left\|\tilde{\pi}_{s x x}\right\|_{p}^{p}+\left\|\tilde{f}_{s}\right\|_{p}^{p}+\left\|\tilde{g}_{s}\right\|_{1, p}^{p}\right] d s+E\left\|\pi_{0}\right\|_{2-2 / p, p}^{p} \\
\leq n+E\left\|\pi_{0}\right\|_{2-2 / p, p}^{p}<\infty .
\end{gathered}
$$

Hence, $\tilde{\pi} \in \mathcal{H}_{p}^{2}(\mathcal{F} ., P, \tau(n))$. By the above mentioned uniqueness, $\tilde{\pi}_{t}=\bar{\pi}_{t}$ on $(0, \tau(n) \rrbracket$ (a.e.). Since both functions are continuous in $t \in[0, T]$ (Theorem 4.2), we have that $\tilde{\pi}_{t} I_{0<t \leq \tau(n)}$ and $\bar{\pi}_{t} I_{0<t \leq \tau(n)}$ are indistinguishable, and since one of them is $\mathcal{F}_{t}^{y}$-adapted, so is the other. We conclude that $\bar{\pi}_{t} I_{0<t \leq \tau(n)}$ is $\mathcal{F}_{t}^{y}$-adapted, which after letting $n \rightarrow \infty$ yields the result. The lemma is proved.

After having obtained (5.2), we proceed as in Section 3 and obtain (3.8) for any $\varphi \in C_{0}^{\infty}\left(\mathbb{R}^{d_{1}}\right)$. Well-known properties of conditional expectations allows us to replace $\varphi(z)$ with any nonnegative Borel $f(x)$, which leads us to (3.2), to the inequality $\int_{\mathbb{R}^{d}} \bar{\pi}_{t}(x) d x>0$ (a.s.), and further to $(2.10)$.

It only remains to prove that

$$
\int_{\mathbb{R}^{d}} \bar{\pi}_{t}(x) d x>0 \quad \forall t \in[0, T]
$$


(a.s.). To do so it suffices to notice that, by Theorem 4.2 , the function $\int_{\mathbb{R}^{d}} \bar{\pi}_{t}^{p}(x) d x$ is continuous (a.s.) and by the above result it is positive (a.s.) for any $t$. Therefore, it is positive for all $t \in[0, T]$ (a.s.). In particular, (5.4) holds true, which brings the proof of the theorem to an end.

\section{REFERENCES}

[1] I.I. Gikhman, Certain differential equations with random functions, Ukr. Mat. Zh., Vol. 2, 3 (1950), 45-69 (in Russian).

[2] N.V. Krylov, "Controlled diffusion processes", Nauka, Moscow, 1977 in Russian; English translation in Springer, 1980.

[3] N.V. Krylov, Introduction to the theory of diffusion processes, Amer. Math. Soc., Providence, RI, 1995.

[4] N.V. Krylov, On $L_{p}$-theory of stochastic partial differential equations, SIAM J. Math. Anal. Vol. 27 (1996) 313-340.

[5] N.V. Krylov, An analytic approach to SPDEs, pp. 185-242 in Stochastic Partial Differential Equations: Six Perspectives, Mathematical Surveys and Monographs, Vol. 64, AMS, Providence, RI, 1999.

[6] N.V. Krylov and A.Yu. Veretennikov, On explicit formulas for solutions of the stochastic equations, Matematicheski Sbornik, Vol. 100, 2 (1976), 266-284 in Russian; English translation in Math. USSR Sbornik Vol. 29, 2 (1976), 239-256.

[7] N.V. Krylov and A. Zatezalo, Filtering of finite-state time-nonhomogeneous Markov processes, a direct approach, to appear in Applied Mathematics and Optimization.

[8] O.A. Ladyzhenskaia, V.A. Solonnikov, and N.N. Ural'tceva: Linear and quasi-linear equations of parabolic type, Nauka, Moscow, 1967 in Russian; English translation: American Math. Soc., Providence, 1968.

[9] G.M. Lieberman, Intermediate Schauder theory for second order parabolic equations IV: time irregularity and regularity, Diff. and Integ. Eqs., Vol. 5 (1992), 1219-1236,.

[10] O.G. Purtukhija and B.L. Rozovskii, Measure-valued solutions to second order stochastic parabolic equations, in "Statistics and Control of Random Processes", editor A.N. Shiryayev, Nauka, Moscow, 1989, pp. 177-179 (in Russian).

[11] B.L. Rozovskii, Stochastic evolution systems, Kluwer Academic Publishers, Dordrecht, Boston, 1990.

[12] B.L. Rozovskii, A simple proof of uniqueness for Kushner and Zakai equations, , pp. 449-458 in "Stochastic Analysis", Academic Press, Boston, 1991.

[13] V.A. Solonnikov, On boundary value problems for parabolic systems of general type, Proceedings of the Steklov institute of Math in the Academy of Sci of the USSR, Vol. 83, 1965, 1-184, Amer Math Soc, Library of Congress Card 66-26640, 1967.

[14] H. Triebel, Theory of function spaces, Birkhäuser Verlag, Basel-Boston-Stuttgart, 1983.

[15] A. Zatezalo, Filtering of Partially Observable Stochastic Processes, University of Minnesota Ph. D. Thesis, Minneapolis, May 1998.

127 Vincent Hall, University of Minnesota, Minneapolis, MN 55455

E-mail address: krylov@math.umn.edu

514 Vincent Hall, University of Minnesota, Minneapolis, MN 55455

E-mail address: zatezalo@ima.umn.edu 